PROCEEDINGS OF THE

AMERICAN MATHEMATICAL SOCIETY

Volume 125, Number 2, February 1997, Pages 381-385

S 0002-9939(97)03607-1

\title{
EXPLICIT BETTI NUMBERS FOR A FAMILY OF NILPOTENT LIE ALGEBRAS
}

\author{
GRANT F. ARMSTRONG, GRANT CAIRNS, AND BARRY JESSUP
}

(Communicated by Roe Goodman)

AbStract. Betti numbers for the Heisenberg Lie algebras were calculated by Santharoubane in his 1983 paper. However few other examples have appeared in the literature. In this note we give the Betti numbers for a family of $(2 n+1)$ dimensional 2-step nilpotent extensions of $\mathbb{R}$ by $\mathbb{R}^{2 n}$.

\section{INTRODUCTION}

Let $\mathfrak{g}$ denote a finite dimensional nilpotent Lie algebra defined over an arbitrary field $k$. Let $\mathfrak{g}^{*}$ denote the vector space dual to $\mathfrak{g}$ and

$$
\wedge \mathfrak{g}^{*}=\bigoplus_{i \geq 0} \wedge^{i} \mathfrak{g}^{*}
$$

the exterior algebra. The differential $d: \wedge \mathfrak{g}^{*} \rightarrow \wedge \mathfrak{g}^{*}$ is the unique derivation of degree one extending

$$
d x^{*}(a \wedge b)=-x^{*}([a, b])
$$

for each $x^{*} \in \wedge^{1} \mathfrak{g}^{*}$ and $a, b \in \mathfrak{g}$. We calculate the Betti numbers $b_{i}(\mathfrak{g})$ of $\mathfrak{g}$ given by

$$
b_{i}(\mathfrak{g})=\operatorname{dim}\left(H^{i}(\mathfrak{g}, k)\right)
$$

for the Lie algebra cohomology with coefficients in $k$.

For every $n \in \mathbb{N}$, let $\mathfrak{h}_{n}$ denote the $n^{\text {th }}$ Heisenberg Lie algebra. This is the $(2 n+1)$-dimensional 2 -step nilpotent Lie algebra with basis $\left\{x_{1}, \ldots, x_{n}, y_{1}, \ldots, y_{n}, z\right\}$ and non-zero relations $\left[x_{i}, y_{i}\right]=z$ for each $1 \leq i \leq n$. According to Santharoubane [5]

$$
b_{i}\left(\mathfrak{h}_{n}\right)=\left(\begin{array}{c}
2 n \\
i
\end{array}\right)-\left(\begin{array}{c}
2 n \\
i-2
\end{array}\right)
$$

for all $0 \leq i \leq n$ (assuming $\left(\begin{array}{l}p \\ q\end{array}\right)=0$ unless $0 \leq q \leq p$ ). The remaining numbers are given by Poincaré duality.

Recall that the Heisenberg Lie algebras arise as extensions of $\mathbb{R}^{2 n}$ by $\mathbb{R}$. We study a family of $(2 n+1)$-dimensional 2 -step nilpotent extensions of $\mathbb{R}$ by $\mathbb{R}^{2 n}$. For every $n \in \mathbb{N}$, let $\mathfrak{g}_{n}$ denote the Lie algebra with basis $\left\{x_{1}, \ldots, x_{n}, y_{1}, \ldots, y_{n}, z\right\}$ and non-zero relations $\left[z, x_{i}\right]=y_{i}$ for each $1 \leq i \leq n$. Our main result is the following.

Received by the editors April 20, 1994 and, in revised form, August 31, 1995.

1991 Mathematics Subject Classification. Primary 17B56; Secondary 17B30, 22E40.

Key words and phrases. Lie algebra, nilpotent, cohomology.

(C)1997 American Mathematical Society 
Theorem. The $i^{\text {th }}$ Betti number $b_{i}\left(\mathfrak{g}_{n}\right)$ is given by

$$
b_{i}\left(\mathfrak{g}_{n}\right)=\left(\begin{array}{c}
n+1 \\
{\left[\frac{i+1}{2}\right]}
\end{array}\right)\left(\begin{array}{c}
n \\
{\left[\frac{i}{2}\right]}
\end{array}\right)
$$

for all $0 \leq i \leq 2 n+1$, where $[x]$ denotes the integer part of $x$.

\section{Proof of The THEOREM}

We use the holonomy derivation $\theta: \wedge \mathbb{R}^{2 n} \rightarrow \wedge \mathbb{R}^{2 n}$ associated to the extension of Lie algebras, which restricts to $\theta_{i}: \wedge^{i} \mathbb{R}^{2 n} \rightarrow \wedge^{i} \mathbb{R}^{2 n}$, to reduce the theorem to a computation of the dimensions of $\operatorname{ker}\left(\theta_{i}^{m}\right)$. These we calculate by finding and solving a recurrence relation involving $n, i$ and $m$.

Let $\left\{x_{1}^{*}, \ldots, x_{n}^{*}, y_{1}^{*}, \ldots, y_{n}^{*}, z^{*}\right\}$ denote the standard dual basis for $\mathfrak{g}_{n}^{*}$ and consider $\wedge \mathbb{R}^{2 n}=\wedge\left\langle x_{1}^{*}, \ldots, x_{n}^{*}, y_{1}^{*}, \ldots, y_{n}^{*}\right\rangle$. Define $\theta: \wedge \mathbb{R}^{2 n} \rightarrow \wedge \mathbb{R}^{2 n}$ to be the unique derivation of degree zero mapping $x_{i}^{*}$ to 0 and $y_{i}^{*}$ to $x_{i}^{*}$ for each $1 \leq i \leq n$. Let $\theta_{i}$ denote the restriction of $\theta$ to $\wedge^{i} \mathbb{R}^{2 n}$ for each $0 \leq i \leq 2 n$.

Lemma 1. The map $\operatorname{ker}\left(\theta_{i}\right) \oplus\left(\wedge^{i-1} \mathbb{R}^{2 n} / \operatorname{Im}\left(\theta_{i-1}\right)\right) \rightarrow H^{i}\left(\mathfrak{g}_{n}, k\right)$ defined by

$$
(\alpha, 0) \mapsto[\alpha],(0,[\beta]) \mapsto\left[z^{*} \wedge \beta\right]
$$

is a vector space isomorphism.

Proof. Extend $\theta$ to $\wedge \mathfrak{g}_{n}^{*}$ by setting $\theta\left(z^{*}\right)=0$. Observe that $d=z^{*} \wedge \theta$, so the $i$ th cocycle and coboundary spaces are given respectively by

$$
Z^{i}\left(\mathfrak{g}_{n}\right)=\operatorname{ker}\left(\theta_{i}\right) \oplus z^{*} \wedge\left(\wedge^{i-1} \mathbb{R}^{2 n}\right) \text { and } B^{i}\left(\mathfrak{g}_{n}\right)=z^{*} \wedge \operatorname{Im}\left(\theta_{i-1}\right) .
$$

The lemma follows immediately.

Corollary 2. For each $0 \leq i \leq 2 n+1$, the $i^{\text {th }}$ Betti number $b_{i}\left(\mathfrak{g}_{n}\right)$ is given by

$$
b_{i}\left(\mathfrak{g}_{n}\right)=\operatorname{dim}\left(\operatorname{ker}\left(\theta_{i}\right)\right)+\operatorname{dim}\left(\operatorname{ker}\left(\theta_{i-1}\right)\right) .
$$

It remains to determine the dimension of each $\operatorname{ker}\left(\theta_{i}\right)$. Consider

$$
\wedge \mathbb{R}^{2(n+1)}=\wedge \mathbb{R}^{2 n} \otimes \wedge x^{*} \otimes \wedge y^{*}
$$

where $\theta_{1}\left(x^{*}\right)=0$ and $\theta_{1}\left(y^{*}\right)=x^{*}$. Denote $\theta_{i}: \wedge \mathbb{R}^{2(n+1)} \rightarrow \wedge \mathbb{R}^{2(n+1)}$ by $\theta_{i, n+1}$. Let $\theta_{i, n}$ denote the restriction of $\theta_{i, n+1}$ to $\wedge^{i} \mathbb{R}^{2 n}$. A short calculation shows that $\operatorname{ker}\left(\theta_{i, n+1}\right)$ depends on $\operatorname{ker}\left(\theta_{i-1, n}^{2}\right)$ (cf. Lemma 3 below), so to study $\operatorname{ker}\left(\theta_{i, n+1}\right)$ we consider arbitrary powers $\theta_{i, n}^{m}(m \geq 0)$ of $\theta_{i, n}$. Observe that any element $\alpha \in$ $\wedge \mathbb{R}^{2(n+1)}$ can be written in the form $\alpha=a+b \wedge x^{*}+c \wedge y^{*}+d \wedge x^{*} \wedge y^{*}$ for some $a, b, c, d \in \wedge \mathbb{R}^{2 n}$. It is easy to verify that such elements belong to $\operatorname{ker}\left(\theta_{i, n+1}^{m}\right)$ if and only if

$$
\theta_{i, n}^{m}(a)=0, \theta_{i-1, n}^{m}(b)+m \theta_{i-1, n}^{m-1}(c)=0, \theta_{i-1, n}^{m}(c)=0 \text { and } \theta_{i-2, n}^{m}(d)=0 .
$$

Lemma 3. The map

$$
\phi_{i, n+1}^{m}: \operatorname{ker}\left(\theta_{i, n}^{m}\right) \oplus \operatorname{ker}\left(\theta_{i-1, n}^{m+1}\right) \oplus \operatorname{ker}\left(\theta_{i-1, n}^{m-1}\right) \oplus \operatorname{ker}\left(\theta_{i-2, n}^{m}\right) \rightarrow \operatorname{ker}\left(\theta_{i, n+1}^{m}\right)
$$

defined by

$$
\phi_{i, n+1}^{m}(a, b, c, d)=a+b \wedge x^{*}+\left(c-(1 / m) \theta_{i-1, n}(b)\right) \wedge y^{*}+d \wedge x^{*} \wedge y^{*}
$$

is a vector space isomorphism. 
Proof. It is easily checked that $\operatorname{Im}\left(\phi_{i, n+1}^{m}\right) \subseteq \operatorname{ker}\left(\theta_{i, n+1}^{m}\right)$. Furthermore the map is clearly injective. To demonstrate surjectivity, suppose we choose an arbitrary element $\alpha=a+b \wedge x^{*}+c \wedge y^{*}+d \wedge x^{*} \wedge y^{*} \in \operatorname{ker}\left(\theta_{i, n+1}^{m}\right)$. Then

$$
\theta_{i, n}^{m}(a)=0, \theta_{i-1, n}^{m+1}(b)=0, \theta_{i-2, n}^{m}(d)=0 \text { and } \theta_{i-1, n}^{m-1}\left(c+(1 / m) \theta_{i-1, n}^{m}(b)\right)=0,
$$

meaning $\phi_{i, n+1}^{m}\left(a, b,\left(c+(1 / m) \theta_{i, n}^{m}(b)\right), d\right)=\alpha$.

Corollary 4. Let $K(n, i, m)=\operatorname{dim}\left(\operatorname{ker}\left(\theta_{i, n}^{m}\right)\right)$. Then one has the recurrence

$$
\begin{aligned}
K(n+1, i, m)= & K(n, i, m)+K(n, i-1, m+1) \\
& +K(n, i-1, m-1)+K(n, i-2, m) .
\end{aligned}
$$

The following boundary conditions are deduced directly from the definition of $\theta$ (condition (3) assumes $\theta^{0}$ is the identity map):

$$
\begin{aligned}
K(0, i, m) & = \begin{cases}0, & \text { if } i \geq 0, m=0 \text { or } i \geq 1, m \geq 1, \\
1, & \text { if } i=0, m \geq 1 .\end{cases} \\
K(n, 0, m) & = \begin{cases}0, & \text { if } m=0, \\
1, & \text { if } m \geq 1 .\end{cases} \\
K(n, 1, m) & = \begin{cases}0, & \text { if } m=0, \\
n, & \text { if } m=1, \\
2 n, & \text { if } m \geq 2 .\end{cases} \\
K(n, i, 0) & =0, \text { for any } n \geq 0 \text { and } i \geq 0 .
\end{aligned}
$$

Notice that the $K(n, i, m)$ 's are uniquely determined by induction on $n$, using the recurrence relation along with these conditions. To facilitate a solution we extend consideration to negative $i$ and $m$ using boundary conditions (2), (3) and (4). A short calculation shows that this amounts to setting $K(n, i, m)=0$ for negative $i$ and $K(n, i,-m)=-K(n, i, m)$ for $m \geq 0$.

Lemma 5. For all $n \geq 0$ and all $i, m \in \mathbb{Z}$, we have

$$
K(n, i, m)=\sum_{p=0}^{n}\left(\begin{array}{c}
n \\
i-p
\end{array}\right)\left(\begin{array}{l}
n \\
p
\end{array}\right) \operatorname{Sign}(m-i+2 p),
$$

where Sign is the usual sign function.

Proof. If we can prove that

$$
K(n, i, m)=\sum_{p=0}^{n} \sum_{q=0}^{n}\left(\begin{array}{l}
n \\
p
\end{array}\right)\left(\begin{array}{l}
n \\
q
\end{array}\right) K(0, i-p-q, m-p+q),
$$

then we obtain expression (5) from boundary condition (1). To prove (6) we proceed by induction on $n$. In the case $n=0$ the result is trivial. The induction step is a straightforward matter of using the recurrence relation and the inductive assumption, along with the binomial identity

$$
\left(\begin{array}{c}
n+1 \\
i
\end{array}\right)=\left(\begin{array}{c}
n \\
i
\end{array}\right)+\left(\begin{array}{c}
n \\
i-1
\end{array}\right)
$$

To determine the Betti numbers we are concerned with the case $m=1$. In this case (5) reduces to the following. 
Corollary 6. We have

$$
K(n, i, 1)=\left\{\begin{array}{l}
\left(\begin{array}{c}
n \\
i / 2
\end{array}\right)^{2}, \text { if } i \text { is even, } \\
\left(\begin{array}{c}
n \\
(i+1) / 2
\end{array}\right)\left(\begin{array}{c}
n \\
(i-1) / 2
\end{array}\right), \text { if } i \text { is odd } .
\end{array}\right.
$$

Finally by Corollary 2, one has

$$
b_{i}\left(\mathfrak{g}_{n}\right)=K(n, i, 1)+K(n, i-1,1)
$$

for all $0 \leq i \leq 2 n+1$. The theorem now follows.

\section{REMARKS}

Figure 1 shows the Betti numbers of the Heisenberg Lie algebras in the cases $n=5,10$ and 40 . These curves have been normalized by dividing by the maximum Betti number in each case. In Figure 2 the analogous curves are depicted for the Lie algebras $\mathfrak{g}_{n}$. The curves for the Heisenberg Lie algebras display an "M" shape, which begins with $n=4$ and becomes more pronounced with larger $n$. By comparison, the distribution of the Betti numbers for $\mathfrak{g}_{n}$ forms a well behaved "Bell" shape for any $n$.

According to Dixmier's theorem [2], one has $b_{i} \geq 2$, for all $0<i<\operatorname{dim} \mathfrak{g}$. However, as Figures 1 and 2 show, this bound is often very weak. One may wonder whether it is true that $b_{i} \geq b_{1}$ for all $0<i<\operatorname{dim} \mathfrak{g}$. This is easy to prove in general for $i=2$, and is easily verified for all $i$ for the families $\mathfrak{h}_{n}$ and $\mathfrak{g}_{n}$.

Lastly notice the theorem is actually valid for some fields of non-zero characteristic. Indeeed Lemma 3 only requires division by elements $m \in k$ less than or equal to the degree of nilpotency of $\theta$. This imposes the obvious restrictions on the characteristic of $k$. By Nomizu's Theorem [4], the cohomology of $\mathfrak{g}_{n}$ over the reals is isomorphic to the cohomology of the nilmanifold $G_{n} / \Gamma_{n}$, where $G_{n}$ is the simply connected Lie group with Lie algebra $\mathfrak{g}_{n}$, and $\Gamma_{n}$ is a uniform lattice, with generators $a_{1}, \ldots, a_{n}, b_{1}, \ldots, b_{n}, c$ and nonzero commutators $c a_{i} c^{-1} a_{i}^{-1}=b_{i}$, for each $1 \leq i \leq n$. The referee has kindly remarked that the cohomology of $\mathfrak{g}_{n}$ over the rationals and over appropriate extensions of the integers is isomorphic to the cohomology of the group $\Gamma_{n}$. The isomorphism over the rationals follows since $\left(\wedge \mathfrak{g}_{n}^{*}, d\right)$

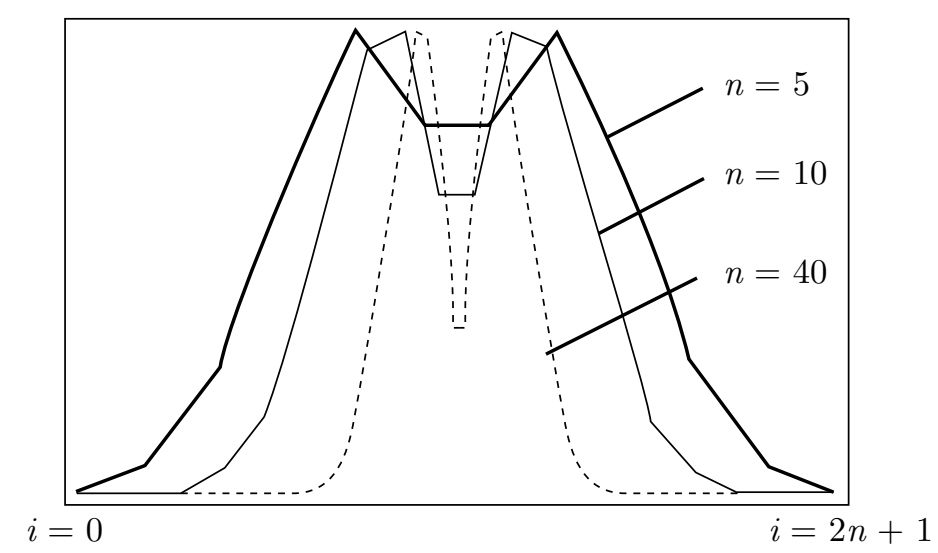

Figure 1. Betti numbers for the Heisenberg Lie algebras 


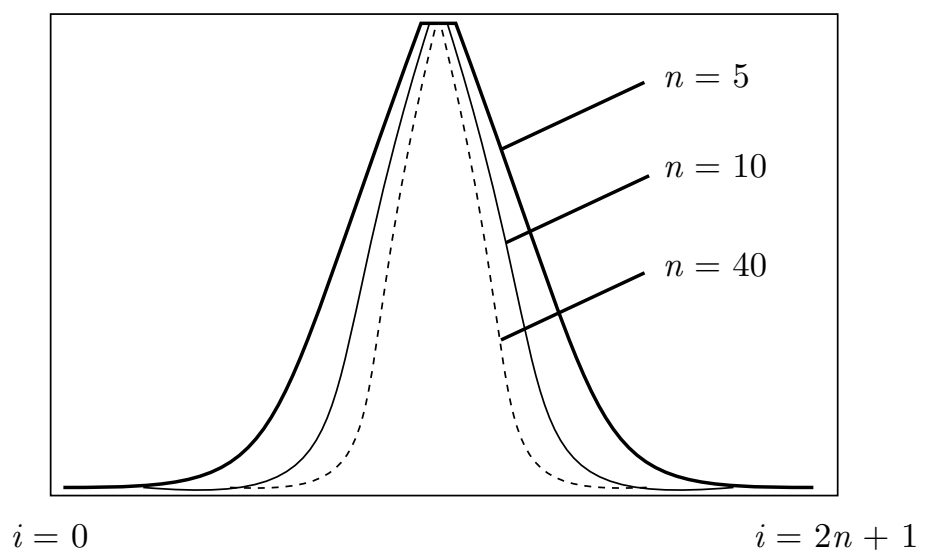

FiguRE 2. Betti numbers for the $\mathfrak{g}_{n}$ 's

is the 1-minimal model for $\Gamma_{n}$ (see [3]); for extensions of the integers obtained by inverting primes in a finite set, the isomorphism follows from [1].

\section{REFERENCES}

1. B.Cenkl and R.Porter, Cohomology of nilmanifolds, Algebraic Topology-Rational Homotopy (Y.Felix, eds.), Lecture Notes in Mathematics, vol. 1318, Springer-Verlag, Berlin and New York, 1988, pp. 73-86. MR 89i:57018

2. J.Dixmier, Cohomologie des algèbres de Lie nilpotentes, Acta Sci. Math. Szeged 16 (1955), 246-250. MR 17:645b

3. P.Griffiths and J.Morgan, Rational Homotopy Theory and Differential Forms, Progress in Mathematics, vol. 16, Birkhäuser, Boston, Basel, Stuttgart, 1981, pp. 73-86. MR 82m:55014

4. K.Nomizu, On the cohomology of compact homogeneous spaces of nilpotent Lie groups, Annals of Math. 59 (1954), 531-538. MR 16:219c

5. L.J.Santharoubane, Cohomology of Heisenberg Lie algebras, Proc. Amer. Math. Soc. 87 (1983), 23-28. MR 87b:17010

(G. F. Armstrong and G. Cairns) School of Mathematics, La Trobe University, MelBourne, Australia 3083

E-mail address: matgfa@lure.latrobe.edu.au

E-mail address: matgc@lure.latrobe.edu.au

(B. Jessup) Department of Mathematics, University of Ottawa, Ottawa, Canada K1N 6N5

E-mail address: bjessup@sciences.uottawa.ca 